\title{
Heterokaryosis in Nectria haematococca: Complementation Between Mutants Affecting the Expression of Two Differentiated States
}

\author{
By M.-J. DABOUSSI-BAREYRE \\ Laboratoire d'Etude et Exploitation du Polymorphisme végétal associé au C.N.R.S., \\ Faculté des Sciences, 91405 Orsay Cedex, France
}

(Received 2 May 1979)

\begin{abstract}
Certain mycelia of the ascomycete Nectria haematococca show two morphological differentiated states, rings and sectors, which are cytoplasmically transmissible. Two nuclear loci $A$ and $S$, appear to be involved in the control of these differentiated states. Mutations in the A region either prevent ring formation and the synthesis of the corresponding infectious factor (type $a$ ) or modify ring expression (type 58). Using forced heterokaryons, it was demonstrated that locus $A$ corresponds to a single gene which exists in three allelic forms, wild-type, $a$ and 58 .
\end{abstract}

\section{INTRODUCTION}

Mycelia of Nectria haematococca can show two morphological modifications, one ringshaped and the other sectorial. Previous studies (Laville, 1971) showed that these modifications were infectious, spread by anastomoses, and that cell-to-cell propagation occurred by the transmission of specific cytoplasmic factors, independently of the nucleus. These new phenotypes may be perpetuated by large inocula, but can return to the initial form after the action of factors which reduce cell metabolism, such as ageing of the mycelium, inhibitors of protein or nucleic acid synthesis, fragmentation or sporulation (Bouvier \& Laville, 1970).

The new mycelial morphologies are interpreted as differentiated forms which succeed a juvenile form (Chevaugeon, 1968). Using mutants, we have shown that the expression of the two differentiated states is controlled by numerous nuclear genes (Bareyre \& LaillierRousseau, 1972; Daboussi-Bareyre et al., 1979). Two nuclear genes, locus $A$ characterized by 14 mutations and locus $S$ by only one, appear to control the synthesis of the cytoplasmic factors associated with the ring and sector phenotypes, respectively. In addition, genetic analysis has shown that mutations in the A region lead to two types of mutants: type $a$ mutants, unable to express ring differentiation, and type 58 mutants, characterized by a modified expression of ring differentiation (Daboussi-Bareyre et al., 1979).

To verify the hypothesis that locus $A$ is a single gene with three allelic forms, wild-type, type $a$ and type 58 , we have developed a functional complementation test, using heterokaryosis. The present report describes the synthesis of heterokaryons and their use in the analysis of the relationships between the genes controlling the expression of the two differentiated states.

\section{METHODS}

Strains. The Nectria haematococca (Berk. and Br.) Wr. strains used were (i) wild-type exhibiting two differentiated states and (ii) five different mutants affecting the expression of these differentiated states.

Wild-type mycelia arising from uninucleate spores have a dense greyish-white aerial mycelium. Morphological modifications appear randomly in the sub-marginal region of these cultures as small brown 
Table 1. Auxotrophic mutants and their origins

$\overbrace{\text { Strain }}^{\text {Origin }}$

Wild-type $\quad \mathrm{NG}\left(200 \mu \mathrm{g} \mathrm{ml}^{-1}\right)$

Wild-type U.v. (400 J m-2)

$58 \quad \mathrm{NG}\left(200 \mu \mathrm{g} \mathrm{ml}^{-1}\right)$

$789 \quad \mathrm{NG}\left(200 \mu \mathrm{g} \mathrm{ml}^{-1}\right)$

ml.58 U.v. $\left(400 \mathrm{~J} \mathrm{~m}^{-2}\right)$

bio* $\quad \mathrm{NG}\left(200 \mu \mathrm{g} \mathrm{ml}^{-1}\right)$ starvation $(8 \mathrm{~d})$ starvation $(15 \mathrm{~d})$ starvation (30 d)
$2 / 2000$ spores analysed

No. of mutants isolated

$1 / 5200$ spores analysed

$4 / 3800$ spores analysed

$1 / 7500$ spores analysed

$3 / 2400$ spores analysed
Metabolic requirements for growth on MM

Biotin Citrulline

Ornithine

Adenine

Citrulline

Lysine

Ornithine

Citrulline

Methionine

p-Aminobenzoic acid

1 double mutant/1000 survivors

0 double mutants $/ 200$ survivors

0 double mutants $/ 3000$ survivors
Citrulline + biotin bio $\arg 13$

* Isolated during mutagenesis of the wild-type strain.

areas in which hyphal elongation is reduced. Once initiated, they progressively invade the margin of the culture at a rate that determines their shape $\mathbf{2 0}$ times the rate of normal hyphal extension for ring differentiation and twice the rate for sector differentiation. These differentiated states can also be transmitted to juvenile mycelia by inoculations performed with large inocula $(2 \times 2 \mathrm{~mm}$ blocks) taken from rings or sectors of suitable age and placed alongside the growing margin of a young mycelium (Laville, 1967).

The four strains a1, a3, ml.58 and 58 differentiate sectors like the wild-type, but are affected in ring development. The first three cannot produce a ring, either spontaneously or after infection. This absence of expression apparently results from the lack of synthesis of the corresponding infectious factor (DaboussiBareyre et al., 1979). Mutant 58 never forms a ring, but experimental inoculation has demonstrated that the infectious ring factor is multiplied throughout the mycelium. Unlike the wild-type, in which ring factor synthesis occurs only in the margin and greatly depresses growth, this synthesis occurs throughout the mycelium of mutant 58 and is accompanied by the diffusion of a red pigment (Daboussi-Bareyre, 1977). Because of these features, we consider this mutant to be modified for ring differentiation. Each of these four strains carries a mutation affecting the same region of the genome, locus $A$. Mutants a1 and 58 are monogenic, strain $\mathrm{m} 1.58$ bears two undissociable mutations in locus $A$ and strain a3 may include another mutation outside locus $A$ (Daboussi-Bareyre et al., 1979). Mutant 789 forms rings like the wild-type, but never has sectors. The infectious sector factor, however, can exist throughout the mycelium and its synthesis is followed by a red pigmentation. Genetic analysis has shown that this mutation is monogenic and involves a locus (locus $S$ ) which is independent of locus $A$ (Daboussi-Bareyre et al., 1979).

Media. Two complete media were used, potato dextrose (PDA) and $\%$ (w/v) Moser malt extract (ME), both containing $2 \%(\mathrm{w} / \mathrm{v})$ agar. The minimal medium (MM) used was as follows: $\mathrm{MgSO}_{4} .7 \mathrm{H}_{2} \mathrm{O}, 0.5 \mathrm{~g}$; $\mathrm{NaNO}_{3}, 2 \mathrm{~g} ; \mathrm{KCl}, 0.5 \mathrm{~g} ; \mathrm{FeSO}_{4} .7 \mathrm{H}_{2} \mathrm{O}, 0.01 \mathrm{~g}$; glucose, $20 \mathrm{~g}$; agar, $20 \mathrm{~g}$; distilled water, 11 . This medium was supplemented with the appropriate compounds for growth of auxotrophic strains.

Production of auxotrophic mutants. Auxotrophs were induced by nitrosoguanidine treatment (NG) or ultraviolet irradiation (u.v.) as previously described (Daboussi-Bareyre et al., 1979). Auxotrophic mutants were selected according to the 'total isolation' technique of Fincham \& Day (1963). Treated spores were diluted and plated on ME. After 24 to $48 \mathrm{~h}$ incubation, small fragments were taken from each colony under a dissecting microscope and transferred to $\mathrm{ME}$ and $\mathrm{MM}$. Clones unable to grow on $\mathrm{MM}$ were retained and their auxotrophy was characterized according to the method of Holliday (1956). These nutritional requirements were confirmed by supplementing MM with single compounds. Only stable mutants unable to grow on supplemented MM were retained (Table 1). Attempts to increase the poor yield of auxotrophs using enrichment techniques like selection with nystatin (Macdonald, 1968) and biotin starvation (Macdonald \& Pontecorvo, 1953) were unsuccessful.

Heterokaryon formation. Auxotrophic mutants were paired on a cellophane membrane by placing together two cotton threads previously dipped in homogenates of each strain. After $48 \mathrm{~h}$ growth on ME, the membrane was transferred to MM. In certain combinations, mycelial growth was observed at the junction of the 


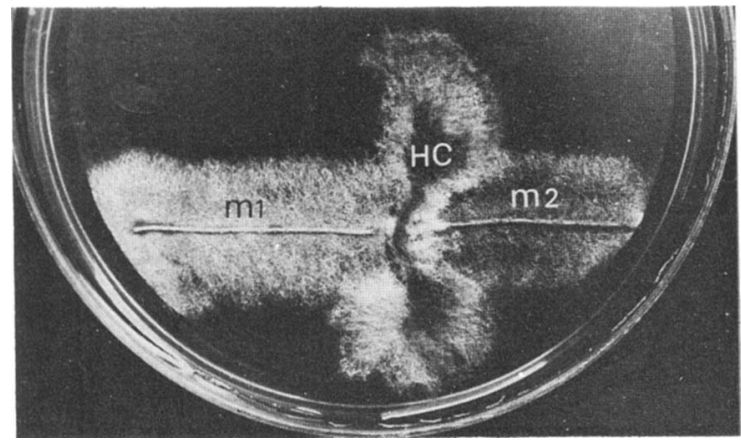

Fig. 1. Pairing of two auxotrophic mutants, $\mathrm{m} 1$ and $\mathrm{m} 2$, showing the formation of a heterokaryotic mycelium (HC) at their junction.

two partners (Fig. 1). Large inocula $(2 \times 2 \mathrm{~mm})$ from this mycelium grew quite well on MM. The formation of prototrophic mycelium did not result from nutritional complementation, since when contact between the two strains was prevented by interposing a cellophane strip there was no growth on MM; this probably required a more intimate association, such as heterokaryosis.

Analysis of prototrophic mycelia. The prototrophic mycelium arising directly from the pairing or from transfer to $\mathrm{MM}$ was gently ground and thoroughly rinsed with distilled water on a sintered glass filter (20 to $40 \mu \mathrm{m}$ porosity) to eliminate conidia. Apical or intercalary hyphal fragments containing no more than four cells were isolated and transferred to different media. Some were transferred to MM to determine the frequency of prototrophic fragments and others were plated on ME to estimate the proportions of the two auxotrophic partners. Three phenotypes could be observed on ME: two types of thalli with fairly smooth mycelia, corresponding to the original homokaryons, which could be distinguished by other criteria (cf. Table 2), and mycelia with a wild-type-like aerial growth, suggesting that the initial fragment contained both types of nuclei. The different nuclear types present in the prototrophic cultures arising from isolated fragments were dissociated and analysed by sampling microconidia.

\section{RESULTS}

\section{Analysis of gene interactions in heterokaryons}

Genetic analyses have shown that among the 14 strains with abnormal ring differentiation, the 10 analysed have a mutation in the same chromosomal region, shorter than the length of one recombination unit, which was called locus $A$ (Daboussi-Bareyre et al., 1979). To determine whether these mutations affected one gene or two adjacent genes, a functional complementation test was performed by creating forced heterokaryons. Because of difficulties encountered in obtaining stable heterokaryons, only six strains were studied by heterokaryosis: the wild-type, one sector mutant and four ring mutants. The combinations involved wild-type and mutant alleles for the determination of dominance relationships; they involved two mutants for allelism tests.

\section{Heterokaryon formation}

Heterokaryosis is not spontaneously established in Nectria haematococca and heterokaryons had to be forced on minimal medium using two strains carrying non-allelic auxotrophic mutations (Pontecorvo, 1953; Parmeter et al., 1963; Caten \& Jinks, 1966). Nutritional deficiencies were induced using $\mathrm{NG}$ or u.v. treatment but it was difficult to recover auxotrophic mutants because of the absence of efficient screening techniques, and the yield was consequently very low, $0.1 \%$ (Table 1 ). The mutants were paired as shown in Fig. 1 and it could be seen that certain combinations of mutants with different metabolic requirements did not necessarily lead to a prototrophic mycelium. Only combinations involving some arginine auxotrophs and one lysine-requiring mutant gave rise to a mycelium which grew well on MM. 
Table 2. Characteristics of the paired strains

\begin{tabular}{|c|c|c|c|}
\hline Strain & Origin & $\begin{array}{c}\text { Mycelial characteristics } \\
\text { on ME }\end{array}$ & $\begin{array}{c}\text { Ability to } \\
\text { express } \\
\text { differentiation } \\
\text { at } 26^{\circ} \mathrm{C} \text { on } \mathrm{ME} \dagger\end{array}$ \\
\hline $\arg 154(58)$ & NG mutagenesis of strain 58 & Smooth, rose-beige & $A * S$ \\
\hline $\arg 154(+)$ & Cross: arg154(58) $\times$ wild-type & Smooth, beige & AS \\
\hline $\arg 154(\mathrm{a} 1)$ & Cross: lys255(a1) $\times \arg 154(58)$ & Smooth, beige & aS \\
\hline $\operatorname{arg13(a1)}$ & Cross: bio $\arg 13(+) \times$ a1 & Velvety, white & $\mathrm{a} \mathbf{S}$ \\
\hline $\operatorname{arg131(789)}$ & NG mutagenesis of strain 789 & Loose, red & AS* \\
\hline $\arg 404(\mathrm{ml} .58)$ & U.v. mutagenesis of strain $\mathrm{ml} .58$ & Smooth, beige & aS \\
\hline lys255(58) & NG mutagenesis of strain 58 & Smooth, violet-beige & $A * S$ \\
\hline lys255(a1) & Cross: arg13(a1) × lys255(58) & Smooth, cream & aS \\
\hline lys255(a3) & Cross: lys $255(58) \times$ a3 & Smooth, cream & aS \\
\hline lys $255(+)$ & Cross: lys $255(\mathrm{a} 3) \times$ wild-type & Smooth, ochre & AS \\
\hline
\end{tabular}

$\uparrow$ AS, Mycelia which differentiate a ring and a sector; aS, mycelia which differentiate a sector but not a ring; As, mycelia which differentiate a ring but not a sector; *, mycelia which have the infectious factor of the modified differentiation.

Properties of associated strains. To prevent ambiguous determination of the interactions among genes controlling differentiation, several strains were created by crossing. They all carried the same auxotrophic markers and thus differed only in the genes being studied (Table 2). Five auxotrophic markers were chosen: one lysine-requirer (lys255) and four arginine-requirers (arg13, arg154, arg131 and $\arg 404)$. These markers did not change the differentiation of the strains (Table 2) but the mycelia of double mutants were smoother than those of the corresponding single prototrophic mutants and their differentiated states appeared with a lower frequency and were less stable.

Associations. Three types of associations were performed. The first involved no interaction between the genes controlling the differentiated states, $+/+$, a1/a1 and 58/58, and enabled us to study the influence of the combination of two mutants differing only in their auxotrophy on the appearance, propagation and maintenance of the differentiated states. The second type included associations in which the wild-type and mutant alleles of a given locus were combined: a1/+, a3/+ and $58 /+$ for locus $A$ and $789 /+$ for locus $S$. The third type involved combinations between mutations affecting either the same locus, such as a1/58, a3/58, $\mathrm{m} 1.58 / 58$, a1/a 3 and $\mathrm{m} 1.58 / \mathrm{a} 1$, or different loci, such as $58+/+789$ and a $1+/+789$.

For all these associations, the presence of a prototrophic mycelium could be seen at the junction line of the two strains. In four cases, $58 /+, 789 /+, 58+/+789$ and a $1 /+789$, two types of reaction were observed at the contact zone and these could be correlated with the levels of activity of genes 58 and 789 . When strains 58 or 789 were paired before the synthesis of ring or sector factors, complementation was shown by the appearance of a mycelium composed of juvenile cells which grew rapidly on MM. But when the production of the infectious factor by strains 58 or 789 had begun before their association in heterokaryosis, each strain induced ring or sector differentiation in its partner (Bareyre \& LaillierRousseau, 1972). This transformation was expressed by the accumulation of a mycelium at the junction line as a swelling, which could not grow on MM.

Analysis of heterokaryotic mycelia. Fragments able to grow on MM were found in most of the pairings analysed, and in all the associations cells combining the properties of both parents could be isolated on complete medium (Table 3). The proportion of heterokaryotic cells was of the order of $1 \%$ or $10 \%$, depending on whether testing was done with transfers to MM or $\mathrm{ME}$, respectively. A reasonable interpretation of this difference is that the unequal proportion of the two nuclear types may result in the rapid isolation of homokaryons which stop growing on MM, whereas multiplication of the minor nuclear population is possible 


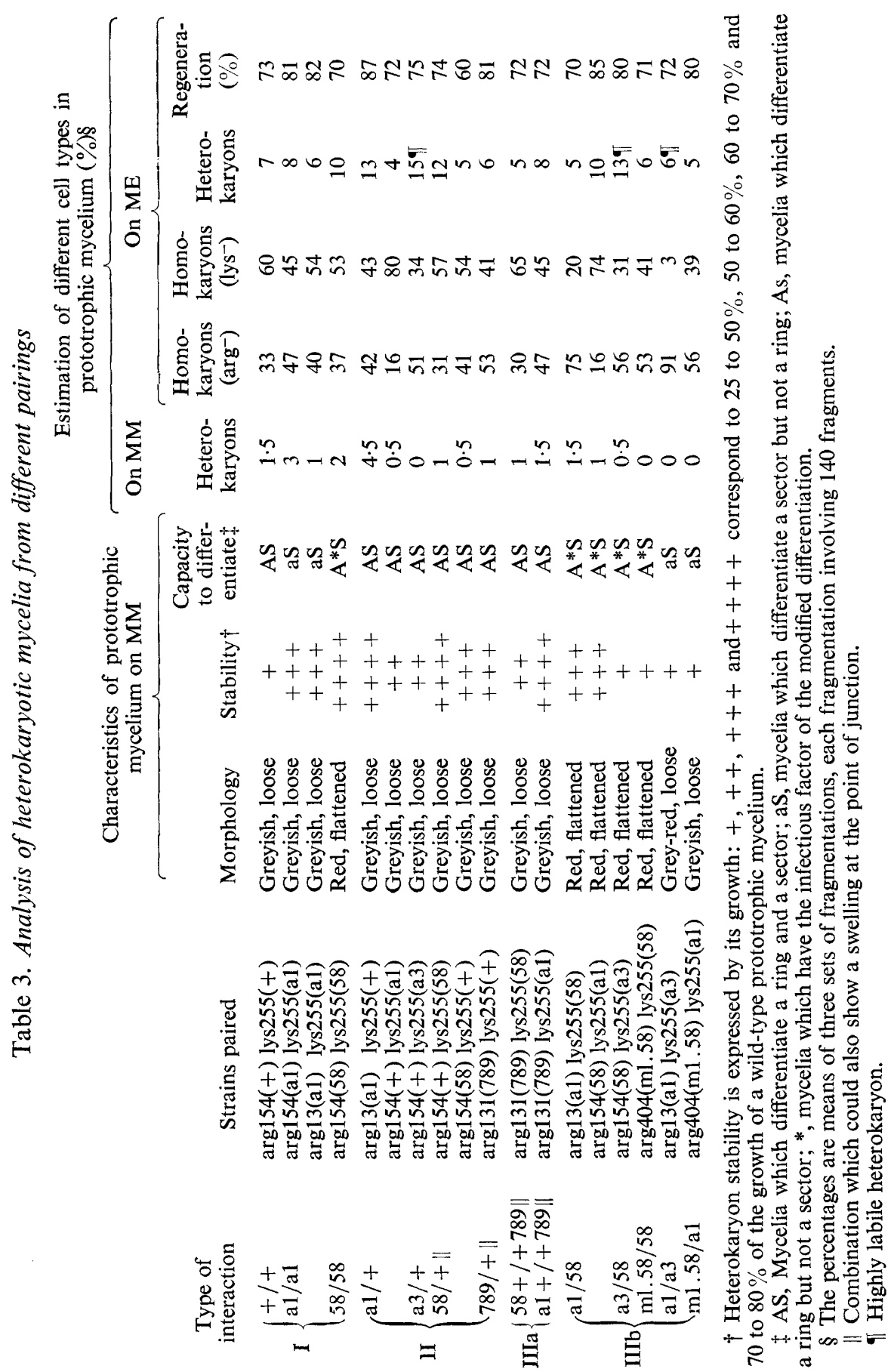



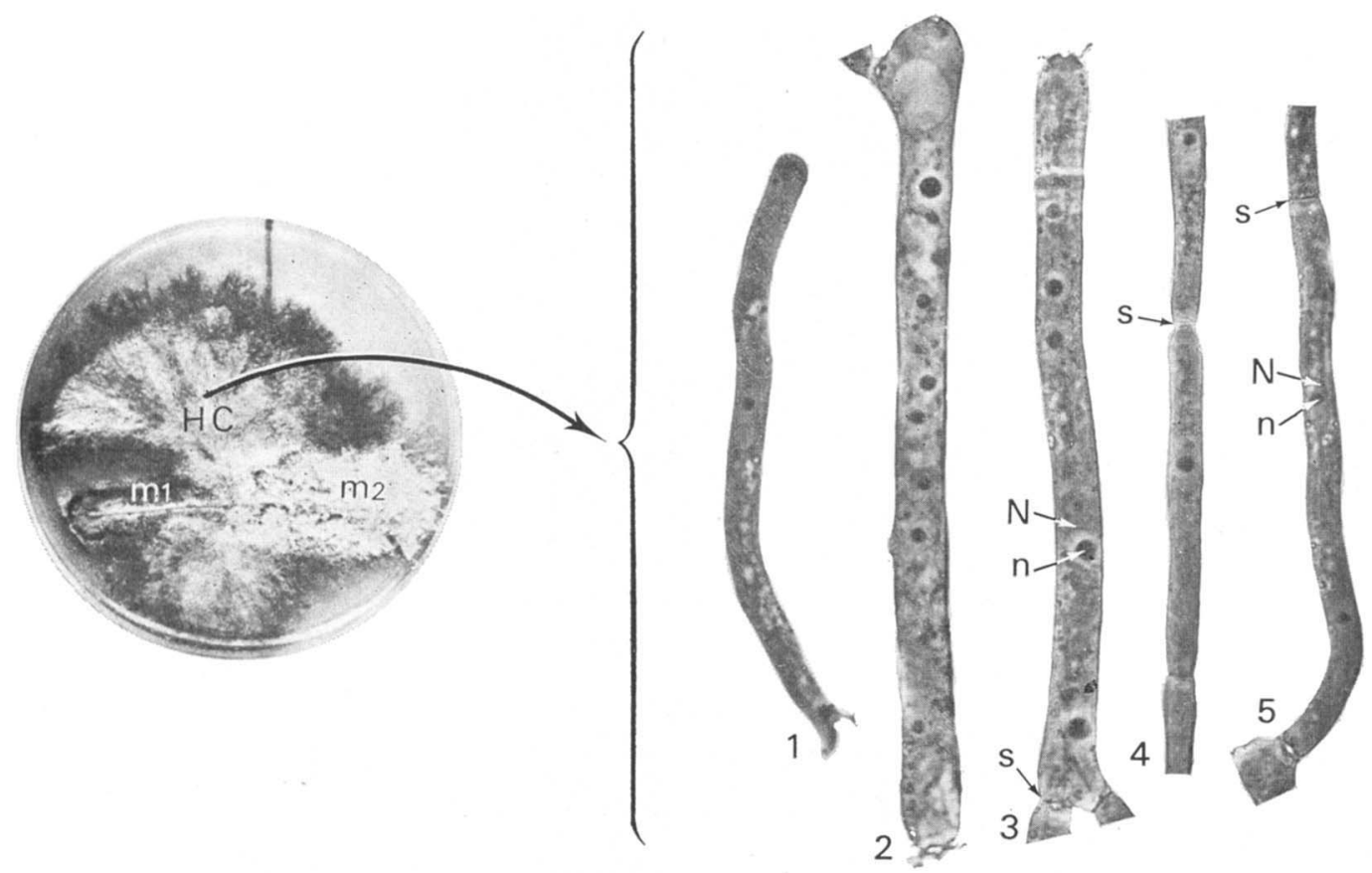

Fig. 2. Examples of cell types forming the heterokaryotic mycelium arising from the combination of mutant lys255(58) [m1] with mutant arg13(a1) [m2]: uninucleate apex (1) or intercalary cells with 8 nuclei (2), 5 nuclei (3), 2 nuclei (5), 1 nucleus (4). HC, Heterokaryotic mycelium; s, septum; $\mathrm{n}$, nucleolus; $\mathrm{N}$, nucleus.

on $\mathrm{ME}$, thus assuring a readjustment of the nuclear balance and allowing complementation. The same influence of culture medium on the proportion of the two cell types has been observed previously, particularly in Fusarium oxysporum (Tuveson \& Garber, 1961; Sanchez et al., 1976).

Phase contrast microscopic observation of the prototrophic mycelium (Fig. 2) revealed the presence of cells containing 2 to 10 nuclei, thus favouring heterokaryosis. We also observed rows of uninucleate cells, suggesting that the heterokaryotic association can be disrupted and thus be limited to a few cells. The very low proportion of heterokaryotic cells in a prototrophic mycelium (about $1 \%$ ) did not prevent its stability. This is particularly true for mycelia arising from the combinations $58 / 58,+/ 58$ and $a 1+/+789$. Their stability was shown by good growth on MM and, in the absence of selection pressure, by the stability of prototrophy at each transfer on ME. The presence of both homokaryons in similar proportions is an important factor for stability; a low proportion of one of the nuclear types leads to instability (a1/a3). However, the stability of the heterokaryotic mycelia appears to be controlled by factors other than the nuclear balance, since certain associations, e.g. $\mathrm{a} 3 /+, \mathrm{m} 1.58 / 58$ and $\mathrm{m} 1.58 / \mathrm{a} 1$, show an approximately equal distribution of nuclei from the two parents and exhibit slow and irregular growth on MM. As has been shown in other organisms (Caten \& Jinks, 1966), there are genes which probably control heterokaryon stability in Nectria also. All the strains used for these pairings originated from the same wildtype strain, but could have accumulated differences, either spontaneously or after mutagenesis, thus causing an instability of the associations. 


\section{Study of the heterokaryons}

The heterokaryotic mycelia were first examined for their pigmentation and texture and their ability to express the two differentiated states, either spontaneously or after experimental inoculation (Table 3).

Does a low proportion of heterokaryotic cells affect the expression of the differentiated states? In type I combinations, both parental strains differ only by non-allelic auxotrophic mutations. The heterokaryon $\arg 154(+) / \operatorname{lys} 255(+)$ has the same phenotype as the wild-type except that the ring is not very stable, as in the parental auxotrophic mutants. In the heterokaryon $\arg 154(\mathrm{a} 1) / \mathrm{lys} 255(\mathrm{a} 1)$ the juvenile morphology is the same as in the wild-type. Sector differentiation is expressed normally, but rings are never formed, either spontaneously or after experimental inoculation, as in mutant a1. Finally, the prototrophic mycelium arising from the combination $\arg 154(58) /$ lys $255(58)$ is red-pigmented, exhibits only the sector, but propagates the infectious ring factor, like strain 58 .

Thus, the nutritional complementation occurring between deficient strains leads to mycelia exhibiting differentiated states similar to those of the original prototrophic strains, except for maintenance of the ring. Lateral propagation of the differentiated states, from cell to cell by anastomosis, is not affected. This suggests that numerous exchanges occur among the three cell types composing the heterokaryotic mycelium. The low number of heterokaryotic cells present in a mosaic prototrophic mycelium should not constitute a major difficulty for the study of intergenic interactions in heterokaryons.

Dominance test. Before testing the allelism of the mutated genes involved in type III associations, it was necessary to show that they were not dominant over their wild-type allele(s). Type II heterokaryons combine wild-type alleles and those altered by the mutations $a 1, a 3,58$ and 789 . The mycelia formed from the pairings a1 $/+, 58 /+$ and $789 /+$ are highly stable and have a wild-type appearance. The irregular growth of the heterokaryotic mycelium arising from the pairing $33 /+$ does not prevent the expression of the two differentiated states, but they are transitory.

The fact that wild-type/mutant heterokaryons have the wild-type phenotype has several implications. (i) The lost capacity to differentiate rings due to mutations $a 1$ and $a 3$ is compensated by the presence of the corresponding function in wild-type nuclei. Genes $a I$ and $a 3$ are thus recessive to their wild-type alleles. (ii) In spite of the preponderance of lys255(58) nuclei, the activity of gene 58 is not expressed in the heterokaryon $\arg 154(+) /$ lys255(58). In a culture derived from a single spore of prototrophic strain 58, the infectious ring factor appears during the third day throughout the mycelium, and the entire culture then becomes red. These features do not appear in a $+/ 58$ heterokaryon. The presence of 58 nuclei is shown by the high frequency of spontaneous rings, which may reach 0.8 in the heterokaryon, compared with 0.2 in the prototrophic wild-type strain. The onset of gene 58 activity is repressed in old cells by the presence of the wild-type allele, which is thus dominant. This repression occurs slightly or not at all in cells of the growing margin, resulting in a high frequency of ring formation. (iii) Similar conclusions could be drawn for the heterokaryon $+/ 789$ : the wild-type allele is dominant over the 789 allele.

Thus, the genes affected by mutations $a 1, a 3$ and 58 (locus $A$ ) and by mutation 789 (locus $S$ ) are recessive to their wild-type alleles. This enables us to perform functional complementation tests.

Allelism test. The existence of complementation indicates that distinct genes are affected by the mutations and a lack of complementation shows that the mutations involve the same gene.

Complementation: type IIIa associations $(58+/+789$ and $\mathrm{a} 1+/+789)$ gave rise to highly stable heterokaryotic mycelia which exhibited rings and sectors (Table 3). Complementation occurred in both loci $A$ and $S$, known to be independent by genetic analysis (Daboussi-Bareyre et al., 1979): each nucleus supplies the function missing in the other. 
Absence of complementation: in type IIIb pairings, involving locus $A$ mutations, mycelia with the properties of the wild-type strain were not observed. Nutritional complementation was not accompanied by complementation between genes affecting ring expression; two different phenotypes were obtained.

Combining the gene modifying ring expression (58) with genes leading to the absence of ring ( $a 1, a 3, m 1.58)$ gave a heterokaryon with the properties of mutant 58 ; it was redpigmented and multiplied the infectious ring factor. This phenotype did not result from a higher number of nuclei bearing the 58 allele (Table 3 ). The latter thus appears fully active in a mixed cytoplasm containing $a 1, a 3$ or $m 1.58$ alleles, as well as in a 58 homokaryon. Unlike the wild-type allele, $a 1, a 3$ and $m l .58$ do not inhibit the activity of 58 .

Combinations of two mutations, each of them leading to the lack of ring expression, e.g. a1/a3 and $\mathrm{m} 1.58 / \mathrm{a} 1$, were unstable (Table 3 ). It is thus difficult to state if the inability of the heterokaryotic mycelium to express a ring results from a non-complementation between the two mutated genes.

\section{DISCUSSION}

The experiments described indicate that heterokaryosis is possible in Nectria haematococca after pairing certain auxotrophic strains. The difficulty of obtaining mycelia with certain combinations suggests that heterokaryon formation is controlled by an incompatibility mechanism. The varying degrees of incompatibility could be due to these systems acting at an early stage during anastomosis (Carr \& Olive, 1959) or later by determining instability or lethality (Wilson et al., 1961; Bernet, 1965). In some pairings, although a heterokaryotic mycelium is formed, relatively few cells ( 1 to $10 \%$ ) are heterokaryotic, probably due to a large number of uninucleate mycelial cells.

Forced heterokaryons are not equally stable on MM. Various degrees of instability could result from a considerable excess of one or the other of the two nuclear types, as well as from other unidentified genetic factors. The formation of stable heterokaryons enabled functional complementation tests to be carried out.

The following conclusions could be drawn from this study:

(i) The alleles mutated at locus $A(58, a 1$ and $a 3)$ and at locus $S(789)$ are recessive.

(ii) Heterokaryons formed from pairings involving mutated alleles of loci $A$ and $S$ have phenotypes similar to the wild-type: complementation occurs satisfactorily between independent $A$ and $S$ loci.

(iii) The heterokaryotic mycelia obtained in combinations of different mutations in locus $A(58, a l, a 3, \mathrm{ml} .58)$ do not exhibit wild-type properties. The instability of certain of these heterokaryons is such that we cannot test for complementation. Nevertheless, the analysis of the properties of the stable combination of alleles 58 and $a l$ of locus $A$ leads to unambiguous conclusions; the heterokaryon shows the properties of mutant 58 .

There are two interpretations of this result; either (a) both mutations affect the same gene and allele 58 is dominant over allele $a 1$ or (b) locus $A$ is composed of two adjacent cistrons and corresponds to a bigenic transcription unit. Mutation al, localized in the gene first transcribed, would prevent the transcription of the adjacent gene, thus having a polar effect. This situation exists in the double mutant $m 1.58$. In the cis position, mutation $a$ prevents the synthesis of the infectious factor, whereas in the trans position, as in the heterokaryon 58/al, this inhibition does not occur. Although the second possibility (b) cannot be ruled out, the simplest hypothesis will be considered in future research, namely that there is only one gene at locus $A$ which can be represented in three allelic forms: wild-type, type al and type 58 .

(iv) The activation of alleles 58 and 789 is prevented when the nuclei containing them are combined with nuclei containing wild-type alleles at loci $A$ and $S$ in cells more than $24 \mathrm{~h}$-old and still undifferentiated. Therefore, the activity of wild-type alleles can repress the onset of the activity of the mutated alleles. 
I thank G. Gerlinger and M. Maugin for expert technical help and Professors J. Chevaugeon and M. Bennoun and Dr D. Parisot for comments on the manuscript.

\section{REFERENCES}

BAREYRe, M.-J. \& LAILlier-Rousseau, D. (1972) Propriétés de nouveaux mutants affectant les variations morphologiques chez le Nectria haematococca. Comptes rendus hebdomadaires des séances de l'Académie des sciences, Série D 274, 3614-3615.

BERNET, J. (1965). Mode d'action des gènes de "barrage" et relation entre l'incompatibilité cellulaire et l'incompatibilité sexuelle chez Podospora anserina. Annales des sciences naturelles Botanique 6, 611.

Bouvier, J. \& LAVILle, E. (1970). Origine et fonction des inducteurs d'états différenciés chez deux Ascomycètes, Physiologie végétale 8, 361-374.

CARr, A. J. H. \& OlIVe, L. S. (1959). Genetics of Sordaria fimicola. III. Cross compatibility among self-fertile and self-sterile cultures. American Journal of Botany 46, 81-91.

CATEN, C. E. \& Jinks, J. L. (1966). Heterokaryosis: its significance in wild homothallic ascomycetes and fungi imperfecti. Transactions of the British Mycological Society 49, 81-93.

Chevaugeon, J. (1968). Etude expérimentale d'une étape du développement du Pestalozzia annulata. Annales des sciences naturelles - Botanique 9, 417432.

DABoussi-BAREYRE, M.-J. (1977). Synthèse et migration de l'information morphogénétique chez Nectria haematococca II. Etude des modalités de l'expression. Physiologie végétale 15, 575-588.

Daboussi-Bareyre, M.-J., LAILlier-Rousseau, D. \& PARIsot, D. (1979). Contrôle génétique de deux états différenciés de Nectria haematococca. Canadian Journal of Botany 57, 1161-1173.

FInchaM, J. R. S. \& DAY, P. R. (1963). Fungal Genetics, p. 45. Oxford: Blackwell Scientific Publications.
Holliday, R. (1956). A new method for identification of biochemical mutants of microorganisms. Nature, London 178, 987.

LAville, E. (1967). Sur une variation contagieuse de l'Hypomyces haematococcus. Comptes rendus hebdomadaires des séances de l'Académie des sciences, Série D 264, 265-267.

Laville, E. (1971). Etude des mécanismes de deux variations contagieuses chez le Nectria haematococca. Thèse de Doctorat d'Etat, Université de Paris-Sud, Centre d'Orsay, France.

MacDonald, K. D. (1968). The selection of auxotrophs of Penicillium chrysogenum with nystatin. Genetical Research 11, 327-330.

Macdonald, K.D. \& Pontecorvo, G. (1953). The genetics of Aspergillus nidulans: starvation technique. Advances in Genetics 5, 159.

PARmeter, J. R., SNyder, W. C. \& Reichle, R. E. (1963). Heterokaryosis and variability in plant pathogenic fungi. Annual Review of Phytopathology 1, 51-76.

PonTeCorvo, G. (1953). The genetics of Aspergillus nidulans: methods of genetic analysis - heterokaryosis. Advances in Genetics 5, 176.

SANChez, L. E., Leary, J. V. \& ENDo, R. M. (1976). Heterokaryosis in Fusarium oxysporum f. sp. lycopersici. Journal of General Microbiology 93, 219-226.

Tuveson, R. W. \& Garber, E. D. (1961). Genetics of phytopathogenicfungi. IV. Experimentally induced alterations in nuclear ratios of heterokaryons of Fusarium oxysporum. Genetics 46, 485-492.

Wilson, J. F., GARNJOBST, L. \& TATUM, E. L. (1961). Heterocaryon incompatibility in Neurospor a crassa - micro-injection studies. American Journal of Botany 48, 299-305. 